\section{Investigación Experimental o Metaanalítica}

Pensar en Movimiento:

Revista de Ciencias del Ejercicio y la Salud

ISSN 1659-4436

Vol. 18, No.2, pp. 1 - 23

Abre $1^{\circ}$ de julio, cierra 31 de diciembre, 2020

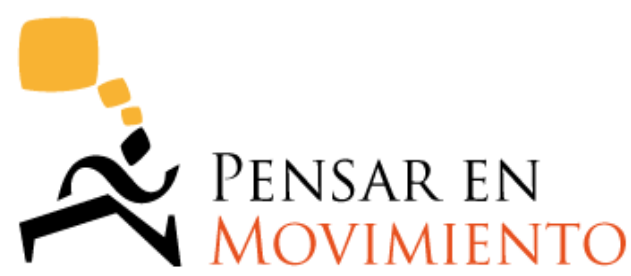

REVISTA DE CIENCIAS DEL EJERCICIO Y LA SALUD

\title{
INTERVENCIÓN DE CAMINATA PARA EMPLEADOS SEDENTARIOS: EFECTO SOBRE LA AUTORREGULACIÓN Y LA AUTO EFICACIA ${ }^{1}$
}

\section{A WALKING INTERVENTION FOR SEDENTARY EMPLOYEES: EFFECTS ON SELF- REGULATION AND SELF-EFFICACY}

\section{INTERVENÇÃO DE CAMINHADA PARA FUNCIONÁRIOS SEDENTÁRIOS: EFEITO SOBRE A AUTORREGULAÇÃO E A AUTOEFICÁCIA}

Mynor G. Rodríguez-Hernández, Ph.D. (D) 2,3 (B-C-D-E) y Danielle Wadsworth, Ph.D. (D) 3 (B-D-E)

mynor.rodriguez@ucr.ac.cr,wadswdd@auburn.edu

${ }^{2}$ Universidad de Costa Rica, San José, Costa Rica

${ }^{3}$ Auburn University, Alabama, Estados Unidos

Envío original: 2020-01-24 Reenviado: 2020-04-03 Aceptado: 2020-04-28

Publicado en versión en español: 2020-11-03

Doi: https://doi.org/10.15517/pensarmov.v18i2.44066

\section{RESUMEN}

Rodríguez-Hernández, M. y Wadsworth, D. (2020). Intervención de caminata para empleados sedentarios: efecto sobre la autorregulación y la auto eficacia. PenSAR EN Movimiento: Revista de Ciencias del Ejercicio y la Salud, 18(1), 1-20. Se diseñó una intervención de caminata de 10 semanas para examinar cómo la actividad física afecta la autorregulación y la autoeficacia

\footnotetext{
${ }^{1}$ Versión traducida al español. Original en inglés disponible en: Rodríguez-Hernández, M., \& Wadsworth, D. (2020). A walking intervention for sedentary employees: effects on self-regulation and self-efficacy. Pensar en Movimiento: Revista de Ciencias del Ejercicio y la Salud, 18(1), e40408. doi: https://doi.org/10.15517/pensarmov.v18i1.40408 $-1-$

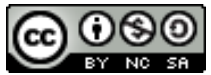


en empleados sedentarios. La intervención fue realizada con 68 participantes, asignados a tres grupos al azar: caminata intermitente, caminata continua o control. La autorregulación, la autoeficacia y la actividad física se midieron al inicio, en la semana 6 y en la semana 11. La actividad de caminata aumentó significativamente $(p<.05)$ con el grupo de caminata continua desde el inicio a la semana $6(p=.033)$. El porcentaje de cambio fue mayor en comparación con el grupo control desde el inicio a la semana $11(p=.042)$. Se observaron mejoras significativas en la autorregulación con el grupo de caminata continua desde el inicio a la semana 6 y a la semana $11(p<.05)$. Sin embargo, la autoeficacia disminuyó desde el inicio a la semana $6(p=.047)$ y a la semana $11(p=.008)$ para todos los grupos. Los empleados sedentarios tendrían más ventajas si se les prescribe un programa de caminata continua, ya que puede mejorar las habilidades de autorregulación. La actividad de caminata intermitente también puede ser un enfoque factible para reducir el comportamiento sedentario; sin embargo, se necesita más investigación para evaluar si los empleados sedentarios pueden o no cumplir con las recomendaciones diarias de actividad física. También es importante revisar en futuras investigaciones el vínculo entre la actividad física y la autoeficacia.

Palabras clave: autorregulación, autoeficacia, comportamiento sedentario, actividad para caminar.

\begin{abstract}
Rodríguez-Hernández, M. \& Wadsworth, D. (2020). A walking intervention for sedentary employees: effects on self-regulation and self-efficacy. PenSAR EN MOVIMIENTO: Revista de Ciencias del Ejercicio y la Salud, 18(1), 1-20. A 10-week walking intervention was designed to examine how physical activity affects self-regulation and self-efficacy in sedentary employees. The intervention was completed by 68 participants randomly assigned to three groups: intermittent walking, continuous walking, or control. Self-regulation, self-efficacy and walking behavior were measured at baseline, week-6, and week-11. Walking activity significantly $(p<.05)$ increased for the continuous walking group from baseline to week-6 $(p=.033)$, the percentage of change was significantly higher compared to the control group from baseline to week-11 $(p=0.042)$. Significant improvements on self-regulation were observed with the continuous group from baseline to week- 6 and week-11 $(p<0.05)$. However, self-efficacy decreased from baseline to week-6 $(p=.047)$ and week-11 $(p=.008)$ for all groups. Sedentary employees may benefit more from a continuous walking program due to enhanced self-regulatory skills. Intermittent walking activity may be also a feasible approach to reduce sedentary behavior, however more research is needed to test whether or not sedentary employees can meet daily physical activity recommendations. It is also important to review in future research, the link between physical activity and self-efficacy.
\end{abstract}


Key words: self-regulation, self-efficacy, sedentary behavior, walking activity.

\section{RESUMO}

Rodríguez-Hernández, M. e Wadsworth, D. (2020). Intervenção de caminhada para funcionários sedentários: efeito sobre a autorregulação e a autoeficácia. PenSAR en Movimiento: Revista de Ciencias del Ejercicio y la Salud, 18(1), 1-120. Desenhou-se uma intervenção de caminhada de 10 semanas para examinar como a atividade física afeta a autorregulação e a autoeficácia em sedentários. A intervenção foi realizada por 68 participantes designados a três grupos aleatórios: caminhada intermitente, caminhada contínua ou controle. A autorregulação, a autoeficácia e a atividade física foram medidas no início, na semana 6 e na semana 11. A atividade de caminhada aumentou significativamente $(p<0,05)$ com o grupo de caminhada contínua do início até a semana $6(p=0,033)$, a porcentagem de troca foi maior em comparação com o grupo controle do início até a semana $11 \quad(p=0,042)$. Foram observadas melhoras significativas na autorregulação com o grupo de caminhada contínua do início até a semana 6 e até a semana $11(p<0,05)$. Porém, a autoeficácia diminuiu do início até a semana $6(p=0,047)$ e até a semana $11(p=0,008)$ para todos os grupos. Os empregados sedentários teriam mais vantagens se thes fosse prescrito um programa de caminhada contínua, pois pode melhorar as habilidades de autorregulação. A atividade de caminhada intermitente também pode ser um enfoque factível para reduzir o comportamento sedentário, no entanto, são necessárias mais pesquisas para avaliar se os empregados sedentários podem ou não cumprir as recomendações diárias de atividade física. É importante também revisar em pesquisas futuras o vínculo entre a atividade física e a autoeficácia.

Palavras-chave: autorregulação, autoeficácia, comportamento sedentário, atividade para caminhar.

A pesar de los beneficios conocidos de la actividad física sobre la salud y la calidad de vida en general, la mayoría de la población adulta de los Estados Unidos es inactiva. De acuerdo con el Centro para el Control y la Prevención de Enfermedades (CDC, 2015), en el 2013, aproximadamente el $80 \%$ de la población de Estados Unidos no cumplió con las recomendaciones de actividad física basadas en medidas auto reportadas. Sin embargo, utilizando medidas objetivas de actividad física, se determinó que alrededor del $95 \%$ de los adultos estadounidenses son inactivos (Troiano et al., 2008). Promover la caminata es una 
estrategia con potencial para aumentar la actividad física. Caminar está relacionado con muchos beneficios para la salud, al tiempo que reduce la posibilidad de lesiones o estrés excesivo (Pelssers et al., 2013; Taylor et al., 2004). Al mismo tiempo, caminar es la actividad física preferida (Williams, Matthews, Rutt, Napolitano, y Marcus, 2008) y una buena alternativa para las personas que son sedentarias y/o que nunca antes han realizado un programa de ejercicio (Ogilvie et al., 2007).

Recientemente, las intervenciones para caminar han cambiado el enfoque de aumentar la actividad física a interrumpir o disminuir el comportamiento sedentario con episodios intermitentes de caminata (Prince, Saunders, Gresty, y Reid, 2014). Se cree que la actividad física ejecutada de forma intermitente tiene beneficios similares para la salud en comparación con la actividad física realizada de forma continua (Bassett, Freedson, y Kozey, 2010; Owen, Healy, Howard, y Dunstan, 2012; Parry, Straker, Gilson, y Smith, 2013; Taylor, 2011). La actividad física intermitente puede requerir menos tiempo (Dunstan, Howard, Healy, y Owen, 2012) y puede aumentar la motivación una vez que las personas se dan cuenta de que es más fácil de realizar (Jakicic, Winters, Lang, y Wing, 1999; Sherwood y Jeffery, 2000) en comparación con sesiones continuas de treinta minutos o más. Además, los períodos cortos de actividad física pueden ser una mejor estrategia para las personas que actualmente no están realizando ningún ejercicio y puede ser más fácil de lograr e incorporar a la vida diaria (Sherwood y Jeffery, 2000), tal como las personas empleadas en oficinas y que son sedentarias la mayor parte de la jornada laboral (Kaewthummanukul y Brown, 2006).

En reportes anteriores se menciona la limitación que han tenido las intervenciones para cambiar los niveles de actividad física a lo largo del tiempo, lo cual ha sido relacionado con la falta de cambio en mediadores clave de la actividad física continuada y la participación en la caminata, como lo son la autorregulación y la autoeficacia (Williams y French, 2011). Si los mediadores clave no se consideran parte de la intervención para mejorar los niveles de actividad física, es poco probable que la actividad física y el hábito de caminar se mantengan (Dishman et al., 2005; Jung y Brawley, 2013).

La autorregulación de la actividad física requiere prestar atención a las propias capacidades, entre ellas, la capacidad de modular los pensamientos, los afectos, el comportamiento o la atención mediante mecanismos de control cognitivo (Buckley, Cohen, Kramer, McAuley, y Mullen, 2014; Karoly, 1993). Algunos aspectos clave para la autorregulación positiva y la adherencia a una conducta físicamente activa son el autocontrol y el manejo del tiempo (Fletcher, Behrens, y Domina, 2008). En una meta-regresión, Michie et al. (2009) establecieron que las intervenciones que buscan un cambio de comportamiento y que usan diferentes herramientas de automonitoreo, producen efectos positivos en los resultados de la adherencia a la actividad física.

Para caminar, se suele utilizar un podómetro y/o dispositivos de muñeca para medir los niveles de actividad física. Estudios previos indican que las personas que realizan un monitoreo de los pasos diarios aumentaron significativamente la actividad física en aproximadamente un 
$27 \%$, en comparación con la evaluación inicial (Bravata et al., 2007). En estudios cualitativos, las personas informaron que los contadores de pasos les ayudaron a aumentar la actividad física, debido a la concientización de los pasos caminados y al establecimiento de objetivos motivadores y significativos al poder ver los pasos dados por día (Lauzon, Chan, Myers y TudorLocke, 2008). En un meta-análisis con 32 estudios (Kang, Marshall, Barreira, y Lee, 2009), los investigadores encontraron que, como herramienta de autocontrol, los podómetros tienen un efecto moderado y positivo sobre el incremento de la actividad física durante el transcurso de las intervenciones, y la meta de caminar 10000 pasos/día es una estrategia eficaz para que las mujeres adultas aumenten la actividad física.

Además de la autorregulación y la autoeficacia, la confianza en que una persona puede realizar un comportamiento específico (Bandura, 1997) está relacionada con la participación continua en el ejercicio. Informes anteriores indican que la autoeficacia es un predictor clave de la adherencia a la actividad física y altos niveles de autoeficacia se relacionan con niveles más altos de participación en la actividad física (Fletcher et al., 2008; Kaewthummanukul y Brown, 2006), del mismo modo, es un factor predictivo para la adopción y el mantenimiento de la actividad física (Sallis et al., 1986; Strachan, Woodgate, Brawley, y Tse, 2005). Además, varios estudios han demostrado que la autoeficacia es un fuerte predictor de cambios en el comportamiento de actividad física en intervenciones a largo plazo (McAuley y Blissmer, 2000; Oman y King, 1998; Sallis et al., 1986). Específicamente, las intervenciones que consideran técnicas como la experiencia vicaria y la retroalimentación logran niveles más altos de actividad física y autoeficacia en comparación con aquellas intervenciones que utilizan la persuasión, el dominio gradual y la identificación de barreras (Ashford, Edmunds, y French, 2010). El uso de estrategias de autoeficacia parece ser efectivo para mejorar la actividad física con el tiempo, y las intervenciones que buscan mejorar el comportamiento de actividad física deben incluir estrategias para producir el conocimiento y la aplicación de esta habilidad (Dishman et al., 2005; Iwasaki et al., 2017).

Aunque la autorregulación y la autoeficacia parecen necesarias para continuar con el ejercicio a largo plazo, existen brechas en la bibliografía sobre cómo los diferentes tipos de actividad física (es decir, continua versus intermitente) afectan la autoeficacia y la autorregulación en adultos sedentarios. Además, los cambios en los niveles de actividad física y su relación con la autorregulación y la autoeficacia no están claros. Por lo tanto, el propósito de este estudio fue examinar el efecto de dos programas diferentes de caminata sobre la autorregulación y la autoeficacia en trabajadores sedentarios de oficina que participaron en un programa de actividad física de 10 semanas

\section{MÉTODO}

El método detallado para este estudio experimental a largo plazo se ha publicado anteriormente (Rodríguez-Hernández y Wadsworth, 2019). Sin embargo, esta sección del estudio agrega aspectos psicológicos que se cree tienen una influencia importante en los 
incrementos de actividad física y la adherencia a lo largo del tiempo. A continuación, se describe el método de forma breve.

\section{Participantes}

Siguiendo las recomendaciones de Cohen sobre una muestra representativa para el análisis del comportamiento tal como la actividad física (cálculo conservador a1 $=.05, r=.30 \mathrm{y}$ potencia $=.80$, con un tamaño de muestra deseable de 68), se asignaron al azar 84 sujetos (según IMC inicial y sexo) a uno de los tres grupos para completar una intervención de 10 semanas, la cual constó de dos protocolos de caminata; caminata intermitente y caminata continua. Un tercer grupo sirvió como grupo control y no recibió prescripción de ejercicio ni entrenamiento para la autorregulación. La figura 1 muestra el diseño de la intervención. Dieciséis participantes se retiraron del estudio durante las primeras semanas de la intervención y los datos se eliminaron de todos los análisis. Por lo tanto, el tamaño de la muestra final fue de 22 participantes para el grupo continuo, 24 para el grupo intermitente y 22 para el grupo de control.

Este protocolo fue aprobado por el Comité de Revisión Institucional de la Universidad de Auburn y siguió los estándares establecidos por la última revisión de la Declaración de Helsinki. Cada participante firmó un consentimiento informado por escrito y completó el Cuestionario de preparación para la actividad física (PAR-Q) antes de participar. 


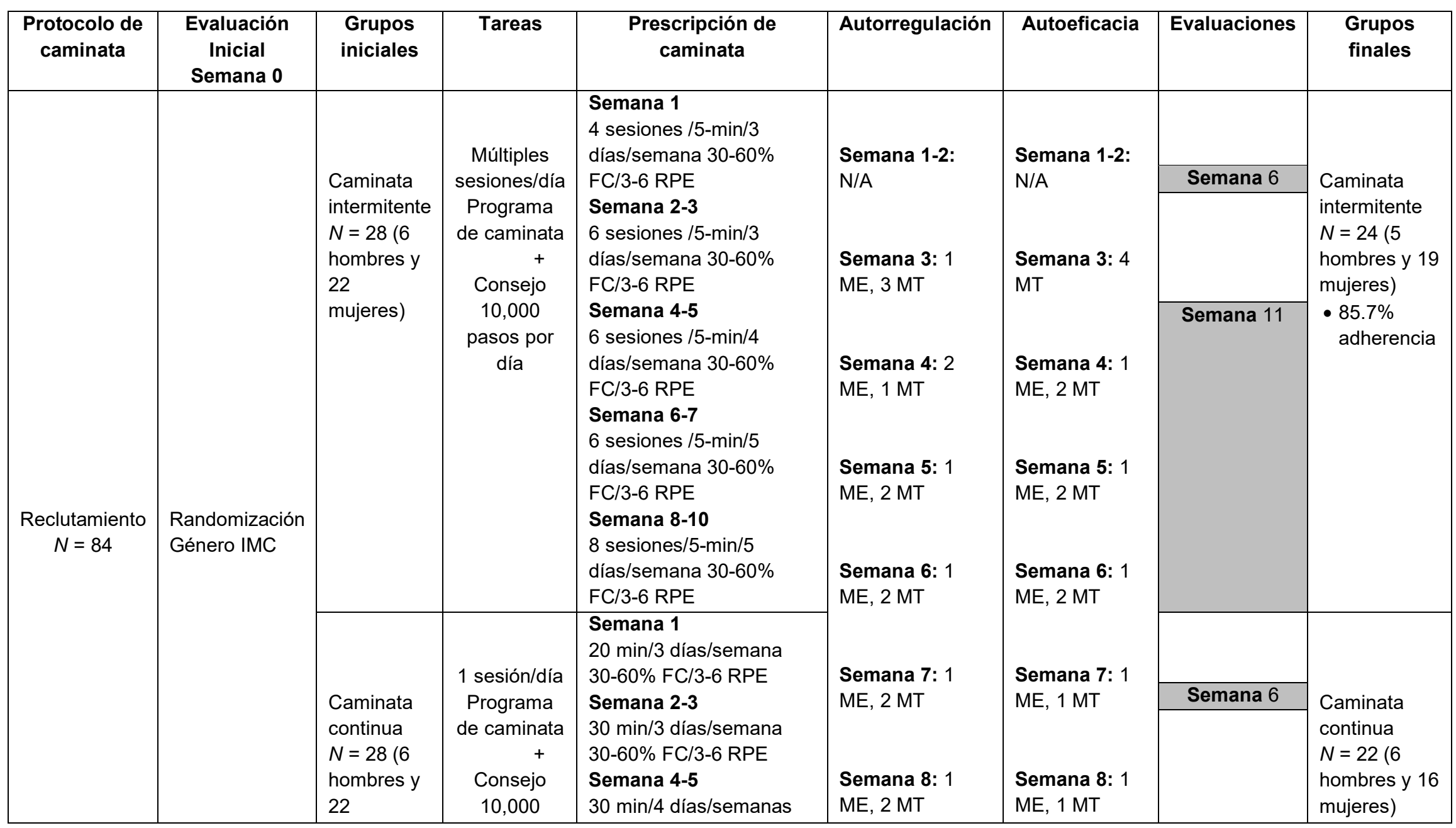

$-7-$ 


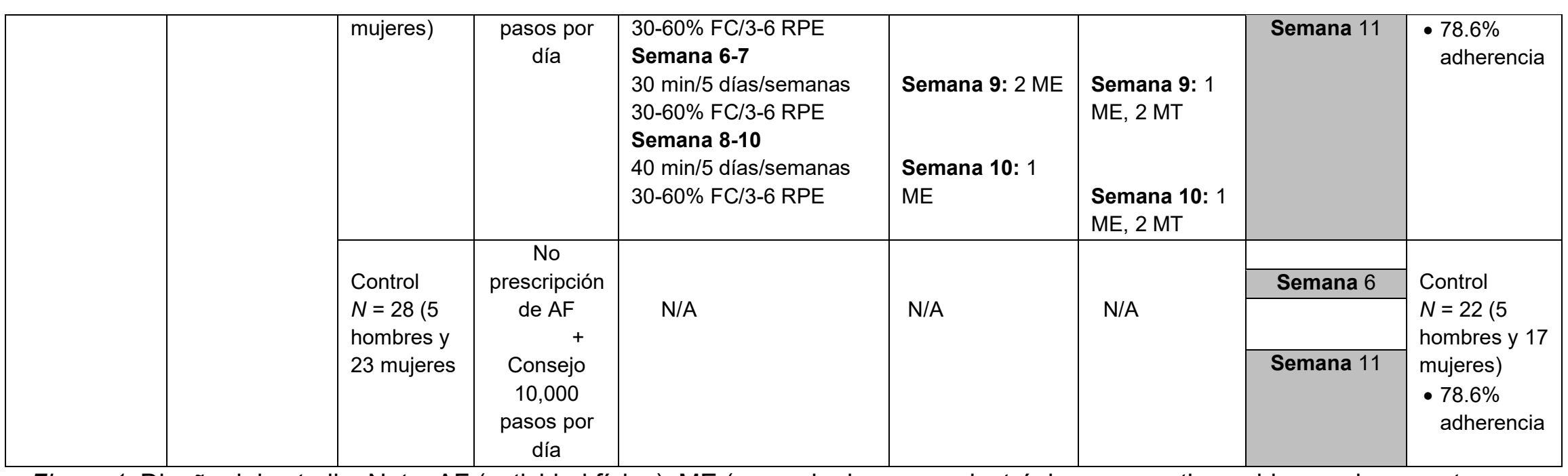

Figura 1. Diseño del estudio. Nota. AF (actividad física), ME (mensaje de correo electrónico que contiene videos o documentos con fines formativos), MT (mensajes de texto), RPE (escala de percepción de esfuerzo), FC (frecuencia cardiaca). Fuente: elaboración propia. 


\section{Procedimientos}

En el punto de partida, se evaluó la autorregulación y la autoeficacia de los participantes a través de cuestionarios y luego se distribuyeron al azar, según el sexo y el IMC, a uno de los tres grupos. Se asignó a cada participante un acelerómetro de muñeca MOVband (Grupo DHS, Houston, TX) para ser usado durante toda la intervención. El acelerómetro puede sincronizarse con un software en la nube y así almacenar los datos que va obteniendo el dispositivo.

La prescripción de caminata de 10 semanas para los grupos intermitente y continuo siguió un aumento escalonado en la práctica de caminata durante 10 semanas. Estos dos grupos fueron educados semanalmente con estrategias para mejorar las habilidades de autoeficacia y autorregulación; se hizo a través de mensajes de texto, correos electrónicos y videos dirigidos a variables específicas como se muestra en la tabla 1. Todos los contenidos se vincularon a tácticas para mejorar el control sobre las acciones personales y para mejorar la confianza en sí mismo, con el fin de cambiar el comportamiento de la actividad física diaria. El grupo de control tuvo acceso a la cuenta MOVband, pero no al programa de caminata ni a las estrategias de autorregulación o autoeficacia que se mencionaron anteriormente. 
Tabla 1

Mensajes de texto y correo electrónico que contienen videos dirigidos a la autorregulación y la autoeficacia durante la intervención de 10 semanas

\begin{tabular}{|c|c|c|c|c|}
\hline \multicolumn{5}{|c|}{ Sistema de mensajes y contenido que promueve la autorregulación y la autoeficacia } \\
\hline & \multicolumn{2}{|c|}{ Autorregulación } & \multicolumn{2}{|c|}{ Autoeficacia } \\
\hline & Objetivo & Ejemplo & Objetivo & Ejemplo \\
\hline Semana 1- 2 & N/A & & N/A & \\
\hline Semana 3 & $\begin{array}{l}\text { Establecimiento de } \\
\text { metas }\end{array}$ & $\begin{array}{l}\text { Video: Establecimiento de metas } \\
\text { MT: "Estacione su vehículo más lejos, } \\
\text { tome las escaleras y camine hasta la } \\
\text { oficina de su amigo. Camine cuando vaya } \\
\text { a almorzar. Levántese de su silla con } \\
\text { frecuencia." }\end{array}$ & Inventario personal & $\begin{array}{l}\text { MT: "Haga un inventario de su experiencia } \\
\text { pasada con el ejercicio. Enumere todos los } \\
\text { aspectos positivos y negativos que pueda } \\
\text { recordar" }\end{array}$ \\
\hline Semana 4 & Automonitoreo & $\begin{array}{l}\text { Video: Automonitoreo } \\
\text { MT: “¿Es consciente del tiempo que pasa } \\
\text { estando físicamente inactivo?" }\end{array}$ & Obstáculos & $\begin{array}{l}\text { MT: "Esté atento a los eventos negativos } \\
\text { que pueden retrasarlo en su rutina de } \\
\text { caminar." }\end{array}$ \\
\hline Semana 5 & Manejo del tiempo & $\begin{array}{l}\text { Video: Manejo del tiempo } \\
\text { MT: “¿Está haciendo tiempo para cumplir } \\
\text { con sus recomendaciones de actividad } \\
\text { física?" }\end{array}$ & Experiencia vicaria & $\begin{array}{l}\text { MT: "Busque lo que otros están haciendo } \\
\text { para mantenerse al día con la actividad } \\
\text { física y los beneficios que están } \\
\text { obteniendo." }\end{array}$ \\
\hline Semana 6 & $\begin{array}{l}\text { Prevención de } \\
\text { recaídas }\end{array}$ & $\begin{array}{l}\text { Video: Prevención de recaídas } \\
\text { MT: "Si surge algo inesperado y se siente } \\
\text { como la excusa perfecta para no hacer } \\
\text { ejercicio, ¿puede hacer y aplicar el plan } \\
\text { B?" }\end{array}$ & Persuasión & $\begin{array}{l}\text { MT: "Asegúrese de tener un plan para } \\
\text { mantenerse al día con su receta para } \\
\text { caminar. La inactividad física es realmente } \\
\text { peligrosa para su calidad de vida y su salud } \\
\text { en general" }\end{array}$ \\
\hline Semana 7 & Apoyo social & $\begin{array}{l}\text { Video: Apoyo social } \\
\text { MT: "Siga haciendo actividad física con } \\
\text { sus amigos, familiares, compañeros de } \\
\text { trabajo, etc. Si aún no tiene uno, busque a } \\
\text { alguien con objetivos similares con quien } \\
\text { ejercitarse" }\end{array}$ & Autoevaluación & $\begin{array}{l}\text { MT: "Tómese un momento para pensar } \\
\text { cómo le va con su programa de actividad } \\
\text { física" }\end{array}$ \\
\hline
\end{tabular}




\begin{tabular}{|c|c|c|c|c|}
\hline Semana 8 & Refuerzos & $\begin{array}{l}\text { Video: Refuerzos } \\
\text { MT: "Recompénsese por mantenerse al } \\
\text { día con el programa de actividad física" }\end{array}$ & Motivación & $\begin{array}{l}\text { MT: "Sigue caminando estás haciendo un } \\
\text { gran progreso. ¡Ese es el modo de seguir!" }\end{array}$ \\
\hline Semana 9 & & $\begin{array}{l}\text { Video: Establecimiento de metas, } \\
\text { automonitoreo y manejo del tiempo }\end{array}$ & Tiempo & $\begin{array}{l}\text { MT: “¿Tiene problemas para cumplir con la } \\
\text { prescripción de actividad física? Asegúrese } \\
\text { de ponerlo dentro de sus prioridades. } \\
\text { ¿Trata de separar el tiempo para hacer } \\
\text { ejercicio?” }\end{array}$ \\
\hline Semana 10 & & $\begin{array}{l}\text { Video: Prevención de recaídas, apoyo } \\
\text { social y refuerzos }\end{array}$ & & $\begin{array}{l}\text { MT: "Comparta sus logros con sus amigos, } \\
\text { familiares y compañeros de trabajo. } \\
\text { Muéstreles sus mejoras". }\end{array}$ \\
\hline
\end{tabular}

Nota. MT (mensaje de texto). Fuente: elaboración propia. 
Se les pidió a los participantes que completaran un cuestionario de autorregulación y autoeficacia, nuevamente, en la semana 6 del programa y al final de la intervención de 10 semanas (semana 11). Los datos del acelerómetro de muñeca se controlaron durante la duración del estudio y se utilizaron tanto los de la evaluación inicial como de la semana 6 y la semana 11, para la comparación.

\section{Medidas}

\section{MOVband}

Para realizar un seguimiento de la actividad física diaria, se entregó un dispositivo móvil de muñeca a los tres grupos, que elabora un registro diario durante toda la intervención (MOVband; DHS Group, Houston, TX). Se ha informado que la confiabilidad del acelerómetro MOVband en cinta rodante es $r=.92, p<.02$ (Barkley, Rebold, Carnes, Glickman, y Kobak, 2014) y en actividad física de la vida diaria $r=.974$ (Williamson, Rebold, Carnes, Glickman, y Barkley, 2014). Un software basado en la nube permitió a los participantes iniciar sesión, sincronizar, descargar datos y cargar el dispositivo cada semana.

\section{Autorregulación}

La autorregulación se midió con un cuestionario de 43 ítems (Petosa, 1993), para evaluar el grado en que se utilizan las estrategias de autorregulación con el fin de apoyar la adherencia al ejercicio regular. Este instrumento contiene seis subescalas 1) refuerzos (ítems 24-32), 2) apoyo social (ítems 15-23), 3) establecimiento de metas (ítems 6-14), 4) autocontrol (ítems 1-5), 5) gestión del tiempo (33-36) y 6) prevención de recaídas (ítems 37-43). Todos los elementos se establecen en una escala Likert que va de 1 (nunca) a 5 (muy a menudo). La autorregulación se definió como habilidades utilizadas para llevar a cabo intenciones de ejercicio y superar barreras personales y situacionales. La validez aparente y de contenido se estableció en una revisión de un panel de expertos en dos etapas. La fiabilidad test-retest para el instrumento total se informó como $r=.92, p<.0001$. La consistencia interna para el instrumento total se informó como .88 (alfa de Cronbach). Los valores sumados mínimo y máximo son 43-215. Una puntuación alta indica el uso frecuente de habilidades de autorregulación.

\section{Autoeficacia}

La autoeficacia se definió como el nivel de autoconfianza en la capacidad para cambiar el comportamiento de la actividad física y se evaluó mediante un instrumento de 12 ítems (Sallis, Pinski, Grossman, Patterson, y Nader, 1988). Esta escala consta de dos subescalas: "Resistir la recaída" (cinco elementos; p. ej., Mantén tu programa de ejercicios cuando tu familia te exige más tiempo) y "Hacer tiempo" para hacer ejercicio (siete elementos; p. ej., Levantarte más temprano para hacer ejercicio). El cuestionario se mide con una escala tipo Likert que va del 1 ("Sé que no puedo hacerlo") al 5 (“Sé que puedo hacerlo"), donde las puntuaciones más altas 
indican una mayor autoeficacia. La confiabilidad de la consistencia interna informada osciló entre .83 y .85 en una población en edad universitaria (Sallis et al., 1988). Además, Speck y Looney informaron que la consistencia interna de esta escala fue de .91 en mujeres de mediana edad que participaban en actividad física de intensidad moderada o alta. La confiabilidad de la test-retest del factor fue de .68 (Speck y Looney, 2001). Cuando se correlacionó la puntuación del factor de autoeficacia con los hábitos de actividad física informados, ambas subescalas se correlacionaron significativamente con la actividad vigorosa informada $(r=.32, p<.001)$ (Sallis et al., 1988).

\section{Análisis estadístico}

Los datos fueron analizados con el Paquete Estadístico para Ciencias Sociales versión 24.0. Con una ANOVA de diseño mixto, se examinó el efecto principal a lo largo del tiempo y el efecto principal del tiempo y la interacción del grupo. Entre factores, se examinaron las diferencias entre los grupos, mientras que, dentro de factores, se evaluaron los cambios con el tiempo dentro de cada grupo. Para un efecto principal significativo (es decir, $p<.05$ ), se realizó la prueba post-hoc de corrección de Bonferroni para comparaciones múltiples. Se realizó una prueba $t$ independiente para comparar el porcentaje de cambio de los datos de movimiento medidos por MOVband.

\section{RESULTADOS}

Sesenta y ocho empleados de oficina sedentarios pudieron finalizar el programa. Al inicio del estudio, los grupos no difirieron en el IMC $(p=.279)$.

\section{La actividad física resulta como movimientos}

La Figura 2 muestra el número promedio de movimientos durante las tres semanas seleccionadas para la evaluación y el porcentaje correspondiente de cambio desde la evaluación inicial hasta la semana 6 y la semana 11. 

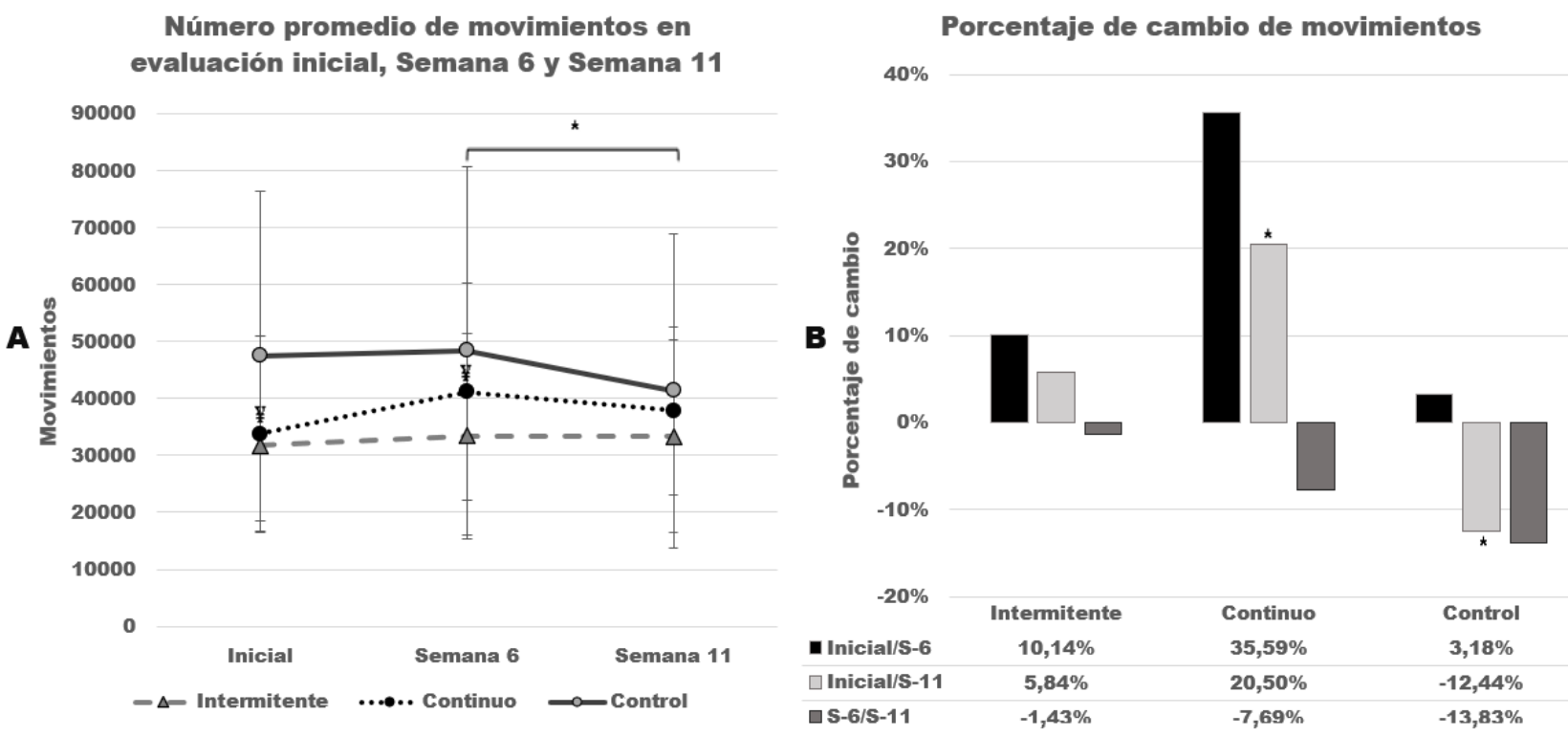

Figura 2. A. Movimientos por grupo, al inicio del estudio, semana 6 y semana $11, p<.05,{ }^{*}=$ Semana 6 a partir de la semana 11. $¥=$ Línea de base desde la semana $6(p<.05)$. B. $p<.05$, porcentaje de cambio por grupo desde el inicio hasta la semana 6 , desde el inicio hasta la semana 11 y desde la semana 6 hasta la semana 11. Fuente: elaboración propia.

Los resultados de la ANOVA de diseño mixto muestran que, para los tres grupos, la actividad física medida por movimientos cambió significativamente durante el transcurso de la intervención con un efecto principal de tiempo $\mathrm{F}_{(2,130)}=4.497, p=.013$ y un efecto de tamaño medio de $\eta^{2}=.065$. La actividad física difirió como efecto principal del tiempo por interacción grupal $F_{(4,130)}=2.526, p=.044$, con un efecto de tamaño medio de $\eta^{2}=.072$. No hubo un efecto principal del grupo sobre la actividad física medida por medio del movimiento $F_{(2,65)}=2.135, p=$ 0.107 . Se encontró una reducción en los movimientos de la semana 6 a la semana $11(p=.014)$ para todos los grupos combinados. La prueba post-hoc de Bonferroni mostró que el grupo de caminata continua aumentó los movimientos desde el inicio hasta la semana $6(p=.033)$, no mostró cambios en los movimientos desde la semana 6 hasta la semana 11 y desde el inicio hasta la semana $11(p>$.05).

La actividad de caminata intermitente y los grupos de control no cambiaron en AF entre las medidas $(p>.05)$, Figura 1A. La prueba t independiente mostró que no hubo diferencias en los movimientos medidos por medio del porcentaje de cambio entre los grupos de caminata intermitente y continua desde el inicio hasta la semana $6(p=169)$, desde el inicio hasta la semana $11(p=.351)$, y de la semana 6 a la semana $11(p=.417)$. Los grupos de caminata intermitente y de control no fueron diferentes entre sí al inicio del estudio hasta la semana 6 ( $p=$

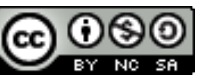

Esta obra está bajo una 
$.527)$, desde el inicio hasta la semana $11(p=.073)$ y desde la semana 6 hasta la semana $11(p$ $=.087)$. Los grupos de caminata continua y control no fueron diferentes entre sí desde el inicio hasta la semana $6(p=.069)$ y desde la semana 6 hasta la semana $11(p=.433)$ pero fueron significativamente diferentes desde el inicio hasta la semana $11(p=.042)$.

\section{Resultados de autorregulación}

El efecto de la intervención sobre la autorregulación se presenta en la Tabla 2

\section{Tabla 2}

Resultados del ANOVA general mixto por grupo para el cuestionario de autorregulación al inicio, a las 6 semanas y a la semana 11

\begin{tabular}{|c|c|c|c|c|c|c|c|c|c|c|c|c|c|c|c|}
\hline & & Inicial & Semana 6 & Semana 11 & Efecto & cipal d & grupo & $\begin{array}{l}\text { Efecto } \mathrm{pr} \\
\text { tiempo }\end{array}$ & cipal de & & $\begin{array}{l}\text { Interacc } \\
\text { grupo }\end{array}$ & de tiem & opor & $\begin{array}{l}\text { Inicial- } \\
\text { Semana } 6 \\
\end{array}$ & $\begin{array}{l}\text { Inicial- } \\
\text { Semana } 11\end{array}$ \\
\hline \multirow{8}{*}{$\begin{array}{l}\text { ANOVA mixta } \\
\text { general }\end{array}$} & & & & & $F$ & $\mathbf{p}$ & $\eta^{2}$ & $\mathrm{~F}$ & $\mathbf{p}$ & $\eta 2$ & $\mathrm{~F}$ & $\mathbf{p}$ & $\eta 2$ & & \\
\hline & Autorregulación & & & & 1.088 & .343 & & 20.040 & $.000^{*}$ & .236 & 8.017 & $.000^{*}$ & .198 & & \\
\hline & Automonitoreo & & & & .788 & .459 & & 18.986 & $.000^{*}$ & .226 & 2.972 & $.022^{*}$ & .084 & & \\
\hline & Establecimiento de metas & & & & .494 & .612 & & 15.087 & $.000^{*}$ & .188 & 5.588 & $.000^{*}$ & .147 & & \\
\hline & Apoyo social & & & & .017 & .983 & & 10.144 & $.000^{*}$ & .135 & 6.463 & $.000^{*}$ & .166 & & \\
\hline & Refuerzos & & & & 2.002 & .143 & & 7.112 & $.001^{*}$ & .099 & 4.197 & $.003^{*}$ & .114 & & \\
\hline & Manejo del tiempo & & & & .053 & .948 & & 32.930 & $.000^{*}$ & .336 & 10.161 & $.000^{*}$ & .238 & & \\
\hline & Prevención de recaídas & & & & 6.332 & $.003^{*}$ & .163 & 33.860 & $.000^{*}$ & .343 & 2.694 & $.034^{*}$ & .077 & & \\
\hline \multirow{7}{*}{ Intermitente } & Autorregulación & $\begin{array}{l}92.33+23.6 \\
0\end{array}$ & $100.25 \pm 22.3$ & $101.38 \pm 29.63$ & & & & & & & & & & & \\
\hline & Automonitoreo & $10.67 \pm 4.28$ & $12.71 \pm 3.90$ & $12.08 \pm 3.68$ & & & & & & & & & & & \\
\hline & Establecimiento de metas & $20.58 \pm 6.71$ & $23.50 \pm 7.23$ & $22.75 \pm 8.03$ & & & & & & & & & & & \\
\hline & Apoyo social & $16.12 \pm 4.93$ & $16.50 \pm 4.47$ & $17.54 \pm 6.37$ & & & & & & & & & & & \\
\hline & Refuerzos & $24.04 \pm 6.69$ & $24.71 \pm 4.89$ & $25.00 \pm 6.73$ & & & & & & & & & & & \\
\hline & Manejo del tiempo & $8.46 \pm 3.06$ & $9.17 \pm 3.28$ & $9.38 \pm 3.70$ & & & & & & & & & & & \\
\hline & Prevención de recaídas & $12.46 \pm 4.15$ & $13.67 \pm 4.56$ & $14.63 \pm 5.11^{*}$ & & & & & & & & & & & $.032^{* *}$ \\
\hline \multirow{7}{*}{ Continuo } & Autorregulación & $\begin{array}{l}75.32+24.6 \\
9\end{array}$ & $112.3 \pm 20.9^{\star}$ & $108.18 \pm 23.05^{\star}$ & & & & & & & & & & $.000^{* *}$ & $.000^{* *}$ \\
\hline & Automonitoreo & $8.09 \pm 3.15$ & $14.05 \pm 4.03^{\star}$ & $12.68 \pm 4.48^{\star}$ & & & & & & & & & & $.000^{* *}$ & $.001^{* *}$ \\
\hline & Establecimiento de metas & $16.68 \pm 6.56$ & $26.86 \pm 6.58^{\star}$ & $25.27 \pm 6.97^{\star}$ & & & & & & & & & & $.000^{* *}$ & $.001^{* *}$ \\
\hline & Apoyo social & $12.59 \pm 4.66$ & $18.82 \pm 5.84^{*}$ & $18.27 \pm 5.47^{*}$ & & & & & & & & & & $.000^{* *}$ & $.001^{* *}$ \\
\hline & Refuerzos & $20.45 \pm 7.81$ & $26.86 \pm 5.99^{*}$ & $26.50 \pm 4.79^{*}$ & & & & & & & & & & $.005^{* *}$ & $.006^{* *}$ \\
\hline & Manejo del tiempo & $6.41 \pm 2.92$ & $10.68 \pm 2.85^{\star}$ & $10.18 \pm 3.23^{*}$ & & & & & & & & & & $.000^{* *}$ & $.001^{* \star}$ \\
\hline & Prevención de recaídas & $11.09 \pm 3.52$ & $15.00 \pm 4.38^{\star}$ & $15.27 \pm 4.30^{*}$ & & & & & & & & & & $.018^{* *}$ & $.001^{* \star}$ \\
\hline \multirow{7}{*}{ Control } & Autorregulación & $87.64 \pm 31.9$ & $89.14 \pm 25.99$ & $92.95 \pm 27.86$ & & & & & & & & & & & \\
\hline & Automonitoreo & $9.50 \pm 3.95$ & $11.45 \pm 4.49$ & $11.18 \pm 5.02$ & & & & & & & & & & & \\
\hline & Establecimiento de metas & $20.45 \pm 9.16$ & $21.09 \pm 8.98$ & $21.64 \pm 8.47$ & & & & & & & & & & & \\
\hline & Apoyo social & $16.41 \pm 6.40$ & $16.23 \pm 6.02$ & $16.77 \pm 5.38$ & & & & & & & & & & & \\
\hline & Refuerzos & $21.55 \pm 7.86$ & $21.23 \pm 5.89$ & $22.59 \pm 7.31$ & & & & & & & & & & & \\
\hline & Manejo del tiempo & $8.05 \pm 3.99$ & $7.23 \pm 2.88$ & $8.09 \pm 2.99$ & & & & & & & & & & & \\
\hline & Prevención de recaídas & $11.68 \pm 3.71$ & $11.91 \pm 3.73$ & $12.68 \pm 4.00$ & & & & & & & & & & & \\
\hline
\end{tabular}

Nota. Los resultados de ANOVA mixtos se presentan en la parte superior de la tabla, los grados de libertad son: efecto principal del grupo (2.65), efecto principal del tiempo $(2,130)$ y tiempo por interacción de grupo $(4,130)$. * $p<.05$, tiempo del efecto principal y tiempo por interacción

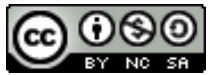


grupal. ${ }^{* *} p<.05$, el grupo de caminata continua mejoró todas las subescalas en la semana 6 y la semana 11. La caminata intermitente mejoró solo la prevención de recaídas de subescala en la semana 11. $¥$ Grupo de control diferente de los grupos intermitente y continuo $(p=.011$ y $p=$ .007 respectivamente). Fuente: elaboración propia.

Los resultados de la ANOVA de diseño mixto mostraron que la autorregulación total cambió como efecto principal del tiempo $\mathrm{F}_{(2,130)}=20.140, p<.001$, con un tamaño de efecto grande de $\eta^{2}=.236$. Al comparar la autorregulación por grupo se encontró una interacción $\mathrm{F}_{(4,130)}=8.017, p<.001$, y un tamaño de efecto grande $\eta^{2}=.198$. La prueba post-hoc de Bonferroni mostró que, para el grupo continuo, la autorregulación general mejoró desde el inicio hasta la semana $6(p<.001)$ y la semana $11(p<.001)$. El grupo de caminata intermitente aumentó en la prevención de recaídas desde el inicio hasta la semana $11(p<.037)$. El grupo de control no cambió la autorregulación general en la semana 6 ni en la semana 11.

\section{Resultados de autoeficacia}

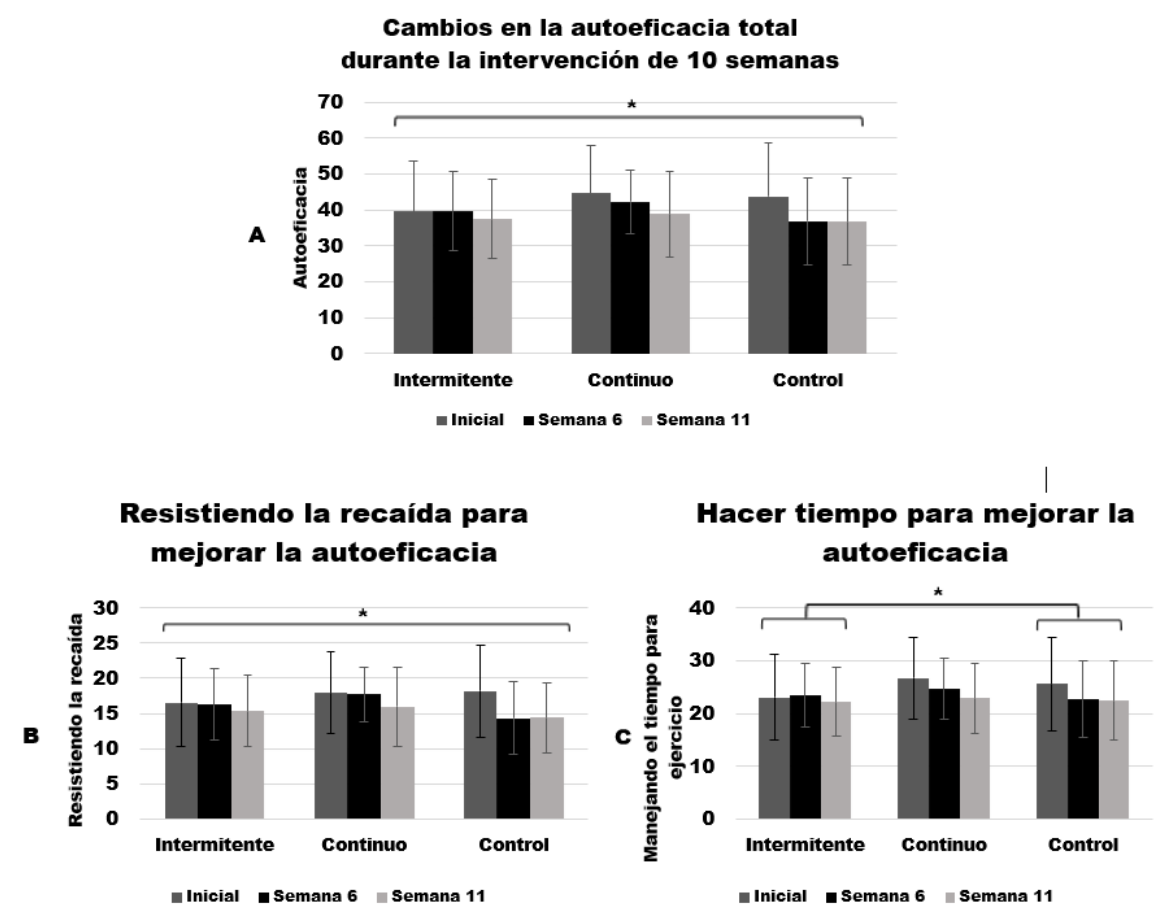

Figura 3. A. Resultados generales de autoeficacia inicial por grupo, prueba de la semana 6 y semana 11. B. Autoeficacia para resistir la recaída por grupo al inicio, prueba de la semana 6 y semana 11. C. Autoeficacia para manejar el tiempo para realizar ejercicio por grupo al inicio, prueba de la semana 6 y semana $11 .{ }^{*} p<.05$. Fuente: elaboración propia.

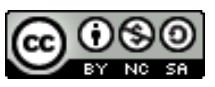

Esta obra está bajo una 
Para la autoeficacia, la figura 3 muestra los resultados de la ANOVA de diseño mixto y demuestra que no hubo efecto del tiempo por interacción de grupo $(F=1.207, p=.312$ ), los grupos no fueron diferentes entre ellos $(F=.571, p=.568)$. No hubo un efecto principal del grupo sobre la autoeficacia $\mathrm{F}_{(2,64)}=.571, p=.568$. La autoeficacia total disminuyó en los tres grupos como efecto principal del tiempo $\left.\mathrm{F}_{(1.821,116.52)}=6.341, p=.003\right)$, con un efecto de tamaño de muestra medio de $\eta^{2}=.090$. La autoeficacia disminuyó significativamente desde el inicio hasta la semana $6(p=.047)$ y desde el inicio hasta la semana $11(p=.008)$. No hubo un efecto principal del tiempo y la interacción del grupo $(F=1.917, p=.112)$ para resistir la recaída. Resistir la recaída como parte de la autoeficacia no cambió como efecto principal del grupo $F_{(2,64)}=.653, p=.524$, pero cambió como efecto principal del tiempo $F_{(2,128)}=7.012, p=.001$, con un efecto de tamaño de muestra medio de $\eta^{2}=.099$. La resistencia a la recaída disminuyó en la semana $6(p=.038)$ y en la semana $11(p=.003)$ en los tres grupos, en comparación con las medidas iniciales. Finalmente, hacer tiempo para el ejercicio no tuvo un efecto principal del grupo $\mathrm{F}_{(2,64)}=.571, p=.568$; sin embargo, se observó un efecto principal del tiempo $\mathrm{F}_{(1.801,115.29)}$ $=4.682, p=.014$, con un efecto de tamaño de muestra medio de $\eta^{2}=.068$. Los tres grupos fueron más bajos en la semana 11 en comparación con el valor inicial $(p=.031)$.

\section{DISCUSIÓN}

El propósito de este estudio fue examinar el efecto de dos programas de caminata diferentes sobre la autorregulación y la autoeficacia para la actividad física en trabajadores de oficina sedentarios después de 10 semanas de intervención. Los resultados mostraron que los individuos dentro de un programa de caminata continua desarrollaron mayores habilidades de autorregulación al comparar con el grupo de control o de caminata intermitente; lo anterior se tradujo en los niveles de actividad física a las 6 y 11 semanas. La autoeficacia disminuyó significativamente durante el transcurso de la intervención para todos los grupos, mostrando una disminución en la confianza para mejorar la conducta de actividad física.

Se definió la autorregulación como el grado en que se utilizan estrategias para apoyar la adquisición de ejercicio regular. Las estrategias como el establecimiento de metas y el autocontrol requieren que el individuo adopte un estado más consciente sobre la voluntad, la planificación, las acciones, el control y la inhibición. Mientras tanto, siguiendo el proceso cognitivo, la autorregulación mejorará cambiando de tareas, aumentando la actividad corporal, mejorando la motivación y desafiando la conducta actual (Bandura, 1991).

Los resultados mostraron varios cambios significativos en la autorregulación, predominantemente en el grupo de caminata continua. Estos hallazgos sugieren que realizar un programa de caminata continua permitió a los individuos autorregular la conducta de caminar mejor que los del grupo de caminata intermitente y el grupo de control. Tanto los grupos de caminata continua como intermitente recibieron la misma intervención móvil que apuntó a las seis habilidades de autorregulación evaluadas; sin embargo, solo la prevención de recaídas, la capacidad de superar las barreras asociadas con el ejercicio, cambió significativamente durante 
el transcurso de la intervención para el grupo intermitente. Esta intervención muestra que la autorregulación se puede cambiar a través de intervenciones móviles, pero que la prescripción de ejercicio para la intervención afecta los cambios en la autorregulación.

Es probable que la demanda de trabajo diario y la conducta sedentaria actual de los participantes del grupo de caminata intermitente interfirieran con la motivación y el control cognitivo para superar las dificultades para cumplir con la prescripción de actividad física. Por lo tanto, los participantes percibieron tareas más desafiantes y menos alcanzables al intercalar varias caminatas cortas todos los días. Este hallazgo está respaldado por un estudio anterior en el que las personas a las que se les prescribieron períodos largos de caminata rápida (30 minutos) participaron en más actividad física que las que se establecieron en un período corto de actividad de caminata (3x10 minutos) (Serwe, Swartz, Hart, y Strath, 2011).

El presente estudio se centró en la autoeficacia mediante la persuasión puntual y la identificación de barreras mediante mensajes de texto y correos electrónicos. Los resultados mostraron que la autoeficacia no mejoró a través de la intervención y, de hecho, la autoeficacia disminuyó significativamente con el tiempo, lo que muestra que la confianza de los participantes para mantenerse al día con la actividad física disminuyó y fueron menos capaces de manejar el tiempo para hacer ejercicio y para resistir las recaídas. Diferentes estudios han sugerido que la autoeficacia cambia con el tiempo, siendo más potente durante las etapas de adopción y más débil durante las etapas de mantenimiento del comportamiento de actividad física (McAuley y Blissmer, 2000; Omán y King, 1998). Además, en estudios previos, los investigadores encontraron que la autoeficacia disminuía con el tiempo con una intervención en línea. Esta disminución en la autoeficacia puede ocurrir porque cuando uno comienza un programa de ejercicios, el nivel de barreras es desconocido y puede aumentar a medida que uno se acerca a la adopción y el mantenimiento (Wadsworth y Hallam, 2010).

Todos los participantes de este estudio pudieron auto-monitorear su comportamiento al caminar durante la duración del estudio. Según investigaciones anteriores, el seguimiento de la actividad física con un rastreador de pasos tiene un impacto importante sobre el comportamiento sedentario en intervenciones que duran al menos 8 semanas (Kang et al., 2009). Además, cuando se les indicó a las mujeres que caminaran 10000 pasos por día, fueron más activas que las que recibieron una prescripción para caminar, con instrucciones para caminar a paso ligero 30 minutos al día todos los días de la semana (Hultquist, Albright, y Thompson, 2005). En el diseño de esta investigación, todos los sujetos recibieron el acelerómetro MOVband y el objetivo de alcanzar 10000 pasos por día. Sin embargo, tener esta herramienta de automonitoreo no se tradujo en cambios en la actividad física para el grupo de control y el grupo intermitente. Solo el grupo continuo pudo mejorar significativamente los movimientos desde el inicio hasta la semana 6 y el porcentaje de cambio fue significativamente diferente del grupo de control en la semana 11.

Además, el grupo de caminata continua mostró un cambio significativamente mayor en los movimientos desde la evaluación inicial hasta la semana 11 en comparación con el grupo de 
control, potencialmente debido a cambios en la autorregulación. Para todos los grupos, hubo una reducción en los movimientos desde la semana 6 hasta el final de la intervención. Con base en información anecdótica de los participantes, el cambio al horario de verano, las vacaciones de Acción de Gracias y otras obligaciones sociales y laborales fueron factores que determinaron una reducción de la actividad física al final de la intervención. Lo anterior está respaldado por una revisión sistemática que mostró que, durante la temporada final del año, las personas son más inactivas (Tucker y Gilliland, 2007).

Los hallazgos acá presentados muestran que, para los empleados sedentarios, un programa estructurado basado en una sola caminata continua puede ser un mejor enfoque para mejorar las habilidades de autorregulación. Se ha demostrado que la mejora en la autorregulación es un mediador clave del cambio y se asocia con niveles más altos de adherencia (Gell y Wadsworth, 2014; Wadsworth y Hallam, 2010). Por lo tanto, un programa de caminata continua puede proporcionar un enfoque más factible para prescribir ejercicio en empleados de oficina sedentarios. La actividad física intermitente puede tener algún impacto positivo en las habilidades de autorregulación; sin embargo, se necesitan más investigaciones para determinar cómo se puede lograr.

\section{LIMITACIONES}

El acelerómetro MOVband permitió observar la actividad física diaria; sin embargo, dado que a todos los participantes se les pidió que sincronizaran el dispositivo usando el teléfono o la computadora, pudieron ver su propia información sobre los movimientos acumulados durante el día, esto pudo haber afectado los resultados del estudio. Los participantes en el grupo de control también pudieron rastrear esta información y podrían haber sido motivados por el acelerómetro y la forma en que este muestra la información. Aunque no se planeó esta característica del diseño, permitió comprender de mejor manera el comportamiento humano y el posible efecto externo que un dispositivo de rastreo puede tener sobre la actividad física, por lo que para futuras pruebas es importante evitar que los participantes tengan acceso a los datos registrados sobre los niveles diarios de actividad física, ya sea en el dispositivo o en el software que se sincroniza en la nube.

\section{AGRADECIMIENTOS}

Queremos agradecer al Dr. Roberts y al Dr. Pascoe por su colaboración en este estudio. Además, nos gustaría agradecer también a los participantes por su logro y dedicación.

TRADUCCIÓN AL ESPAÑOL: Ph.D. Mynor G. Rodríguez-Hernández, Universidad de Costa Rica y Auburn University. 


\section{REFERENCIAS}

Ashford, S., Edmunds, J., y French, D. P. (2010). What is the best way to change self-efficacy to promote lifestyle and recreational physical activity? A systematic review with metaanalysis. British Journal of Health Psychology, 15(2), 265-288. doi: https://doi.org/ $10.1348 / 135910709 \times 461752$

Bandura, A. (1991). Social Cognitive Theory of Self-Regulation. Organizational Behavior and Human Decision Processess, 50(2), 248-287. doi: https://doi.org/10.1016/07495978(91)90022-L

Bandura, A. (1997). Self-efficacy: The Exercise of Control. New York: Freeman.

Barkley, J. E., Rebold, M., Carnes, A., Glickman, E. L., y Kobak, M. (2014). The Validity of a Commercially-available, Low-cost Accelerometer During Treadmill Exercise. Paper presented at the 61st Meeting of the American College of Sports Medicine, Orlando, FL.

Bassett, D. R., Jr., Freedson, P., y Kozey, S. (2010). Medical hazards of prolonged sitting. Exercise and sport sciences reviews, 38(3), 101-102. doi: https://doi.org/10.1097/ JES.0b013e3181e373ee

Bravata, D. M., Smith-Spangler, C., Sundaram, V., Gienger, A. L., Lin, N., Lewis, R., ... Sirard, J. R. (2007). Using pedometers to increase physical activity and improve health: a systematic review. Journal of the American Medical Association, 298(19), 2296-2304. doi: https://doi.org/10.1001/jama.298.19.2296

Buckley, J., Cohen, J. D., Kramer, A. F., McAuley, E., y Mullen, S. P. (2014). Cognitive control in the self-regulation of physical activity and sedentary behavior. Frontiers in Human Neuroscience, 8, 747. doi: https://doi.org/10.3389/fnhum.2014.00747

Centers for Disease Control and Prevention [CDC]. (2015). Behavioral Risk Factor Surveillance System.

Recuperado de

https://www.cdc.gov/chronicdisease/resources/publications/factsheets/brfss.htm

Dishman, R. K., Motl, R. W., Sallis, J. F., Dunn, A. L., Birnbaum, A. S., Welk, G. J., ... Jobe, J. B. (2005). Self-Management Strategies Mediate Self-Efficacy and Physical Activity. American Journal of Preventive Medicine, 29(1), 10-18. doi: https://doi.org/ 10.1016/j.amepre.2005.03.012

Dunstan, D. W., Howard, B., Healy, G. N., y Owen, N. (2012). Too much sitting--a health hazard. Diabetes Research and Clinical Practice, 97(3), 368-376. doi: https://doi.org/10.1016/ j.diabres.2012.05.020

Fletcher, G. M., Behrens, T. K., y Domina, L. (2008). Barriers and enabling factors for work-site physical activity programs: a qualitative examination. Journal of Physical Activity and Health, 5(3), 418-429. doi: https://doi.org/10.1123/jpah.5.3.418

Gell, N. M., y Wadsworth, D. D. (2014). How do they do it: working women meeting physical activity recommendations. American Journal of Health Behavior, 38(2), 208-217. Recuperado de https://www.ncbi.nlm.nih.gov/pmc/articles/PMC4043126/ 
Hultquist, C. N., Albright, C., y Thompson, D. L. (2005). Comparison of walking recommendations in previously inactive women. Medicine and Science in Sports and Exercise, 37(4), 676-683. Recuperado de https://www.ncbi.nlm.nih.gov/pubmed/15809569

Iwasaki, Y., Honda, S., Kaneko, S., Kurishima, K., Honda, A., Kakinuma, A., y Jahng, D. (2017). Exercise Self-Efficacy as a Mediator between Goal-Setting and Physical Activity: Developing the Workplace as a Setting for Promoting Physical Activity. Safety and Health at Work, 8(1), 94-98. doi: https://doi.org/10.1016/j.shaw.2016.08.004

Jakicic, J. M., Winters, C., Lang, W., y Wing, R. R. (1999). Effects of intermittent exercise and use of home exercise equipment on adherence, weight loss, and fitness in overweight women: a randomized trial. Journal of the American Medical Association, 282(16), 1554-1560. doi: https://doi.org/10.1001/jama.282.16.1554

Jung, M. E., y Brawley, L. R. (2013). Concurrent self-regulatory efficacy as a mediator of the goal: Exercise behaviour relationship. Journal of Health Psychology, 18(5), 601-611. doi: https://doi.org/10.1177/1359105313479238

Kaewthummanukul, T., y Brown, K. C. (2006). Determinants of employee participation in physical activity: critical review of the literature. AAOHN Journal, 54(6), 249-261. Recuperado de https://journals. sagepub.com/doi/10.1177/216507990605400602

Kang, M., Marshall, S. J., Barreira, T. V., y Lee, J. O. (2009). Effect of pedometer-based physical activity interventions: a meta-analysis. Research Quarterly for Exercise and Sport, 80(3), 648-655. doi: https://doi.org/10.1080/02701367.2009.10599604

Karoly, P. (1993). Mechanisms of self-regulation: a systems review. Annual Reviews of Psychology, 44, 23-52. doi: https://doi.org/10.1146/annurev.ps.44.020193.000323

Lauzon, N., Chan, C. B., Myers, A. M., y Tudor-Locke, C. (2008). Participant experiences in a workplace pedometer-based physical activity program. Journal of Physical Activity and Health, 5(5), 675-687. doi: https://doi.org/10.1123/ipah.5.5.675

McAuley, E., y Blissmer, B. (2000). Self-efficacy determinants and consequences of physical activity. Exercise and sport sciences reviews, 28(2), 85-88. Recuperado de https://www.ncbi.nlm.nih.gov/pubmed/10902091

Michie, S., Abraham, C., Whittington, C., McAteer, J., y Gupta, S. (2009). Effective techniques in healthy eating and physical activity interventions: a meta-regression. Health Psychology, 28(6), 690-701. doi: https://doi.org/10.1037/a0016136

Ogilvie, D., Foster, C. E., Rothnie, H., Cavill, N., Hamilton, V., Fitzsimons, C. F., ... Scottish Physical Activity Research, C. (2007). Interventions to promote walking: systematic review. British Medical Journal, 334(7605), 1204-1214. doi: https://doi.org/10.1136/b mj.39198.722720.BE

Oman, R. F., y King, A. C. (1998). Predicting the adoption and maintenance of exercise participation using self-efficacy and previous exercise participation rates. American 
Journal of Health Promotion, 12(3), 154-161. doi: https://doi.org/10.4278/0890-117112.3 .154

Owen, N., Healy, G. N., Howard, B., y Dunstan, D. W. (2012). Too Much Sitting: Health Risks of Sedentary Behaviour and Opportunities for Change. Research Digest, 13(3). Recuperado de https://www.apcointl.org/doc/conference-documents/personnel-humanfactor/519-too-much-sitting-health-risks-of-sedentary-behavior-and-opportunities-forchange/file.html

Parry, S., Straker, L., Gilson, N. D., y Smith, A. J. (2013). Participatory workplace interventions can reduce sedentary time for office workers--a randomised controlled trial. PLoS One, 8(11), e78957. doi: https://doi.org/10.1371/journal.pone.0078957

Pelssers, J., Delecluse, C., Opdenacker, J., Kennis, E., Van Roie, E., y Boen, F. (2013). "Every step counts!": effects of a structured walking intervention in a community-based senior organization. Journal of Aging and Physical Activity, 21(2), 167-185. doi: https://doi.org/ 10.1123/japa.21.2.167

Petosa, S. (1993). Use of social cognitive theory to explain exercise behavior among adults (Doctoral Dissertation). Ohio State University.

Prince, S. A., Saunders, T. J., Gresty, K., y Reid, R. D. (2014). A comparison of the effectiveness of physical activity and sedentary behaviour interventions in reducing sedentary time in adults: a systematic review and meta-analysis of controlled trials. Obesity Reviews, 15(11), 905-919. doi: https://doi.org/10.1111/obr.12215

Rodriguez-Hernandez, M. G., y Wadsworth, D. W. (2019). The effect of 2 walking programs on aerobic fitness, body composition, and physical activity in sedentary office employees. PLoS One, 14(1), e0210447. doi: https://doi.org/10.1371/journal.pone.0210447

Sallis, J. F., Haskell, W. L., Fortmann, S. P., Vranizan, K. M., Taylor, C. B., y Solomon, D. S. (1986). Predictors of adoption and maintenance of physical activity in a community sample. Preventive Medicine, 15(4), 331-341. doi: https://doi.org/10.1016/00917435(86)90001-0

Sallis, J. F., Pinski, R. B., Grossman, R. M., Patterson, T. L., y Nader, P. R. (1988). The development of self-efficacy scales for health related diet and exercise behaviors. Health Education Research, 3(3), 283-292. doi: https://doi.org/10.1093/her/3.3.283

Serwe, K. M., Swartz, A. M., Hart, T. L., y Strath, S. J. (2011). Effectiveness of long and short bout walking on increasing physical activity in women. Journal of Women's Health, 20(2), 247-253. doi: https://doi.org/10.1089/jwh.2010.2019

Sherwood, N. E., y Jeffery, R. W. (2000). The behavioral determinants of exercise: implications for physical activity interventions. Annual Review of Nutrition, 20, 21-44. doi: https://doi.org/10.1146/annurev.nutr.20.1.21

Speck, B. J., y Looney, S. W. (2001). Effects of a minimal intervention to increase physical activity in women: daily activity records. Nursing Research, 50(6), 374-378. Recuperado de https://www.ncbi.nlm.nih.gov/pubmed/11725940 
Strachan, S. M., Woodgate, J., Brawley, L. R., y Tse, A. (2005). The relationship of self efficacy and self-identity to long-term maintenance of vigorous physical activity. Journal of Applied Biobehavioral Research, 10, 98-112. doi: https://doi.org/10.1111/j.17519861.2005.tb00006.x

Taylor, A. H., Cable, N. T., Faulkner, G., Hillsdon, M., Narici, M., y Van Der Bij, A. K. (2004). Physical activity and older adults: a review of health benefits and the effectiveness of interventions. Journal of Sports Sciences, 22(8), 703-725. doi: https://doi.org/ 10.1080/02640410410001712421

Taylor, W. C. (2011). Prolonged Sitting and the Risk of Cardiovascular Disease and Mortality. Current Cardiovascular Risk Reports, 5(4), 350-357. doi: https://doi.org/10.1007/s12170-011-0174-4

Troiano, R. P., Berrigan, D., Dodd, K. W., Masse, L. C., Tilert, T., y McDowell, M. (2008). Physical activity in the United States measured by accelerometer. Medicine and Science in Sports and Exercise, 40(1), 181-188. doi: https://doi.org/10.1249/mss.0b013e31815a51b3

Tucker, P., y Gilliland, J. (2007). The effect of season and weather on physical activity: a systematic review. Public Health, 121(12), 909-922. doi: https://doi.org/10.1016/j.puhe. 2007.04.009

Wadsworth, D. D., y Hallam, J. S. (2010). Effect of a web site intervention on physical activity of college females. American Journal of Health Behavior, 34(1), 60-69. doi: https://doi.org/10.5993/AJHB.34.1.8

Williams, D. M., Matthews, C. E., Rutt, C., Napolitano, M. A., y Marcus, B. H. (2008). Interventions to increase walking behavior. Medicine and Science in Sports and Exercise, 40(7 Suppl), S567-573. doi: https://doi.org/10.1249/MSS.0b013e31817c7006

Williams, S. L., y French, D. P. (2011). What are the most effective intervention techniques for changing physical activity self-efficacy and physical activity behaviour--and are they the same? Health Education Research, 26(2), 308-322. doi: https://doi.org/10. 1093/her/cyr005

Williamson, M. L., Rebold, M., Carnes, A., Glickman, E. L., y Barkley, J. E. (2014). The Validity of a Low-cost Accelerometer During Free-living Physical activity. Paper presented at the 61 st Meeting of the American College of Sports Medicine, Orlando, FL.

Participación: A- Financiamiento, B- Diseño del estudio, C- Recolección de datos, D- Análisis estadístico e interpretación de resultados, E- Preparación del manuscrito 